\title{
Safety and efficacy of using advanced electrosurgical bipolar vessel sealing during vaginal hysterectomy in morbidly obese patients: a retrospective cohort analysis
}

\author{
Tolga Karacan, Eser Ozyurek, Lale Susan Wetherilt, Huseyin Kiyak, Salman Yilmaz, Erdal Kaya \\ Istanbul Health Sciences, Kanuni Sultan Suleyman Education and Research Hospital, Istanbul, Turkey
}

\begin{abstract}
Objectives: To assess the safety and efficacy of electrosurgical bipolar vessel sealing during vaginal hysterectomy in morbidly obese patients.

Material and methods: A total of 105 morbidly obese patients who underwent vaginal hysterectomy due to benign gynecologic pathologies between January 2010 and April 2017 were included in the study. Patients were divided into two groups according to whether conventional suture ligation technique $(n=64)$ or electrosurgical bipolar vessel sealing $(n=41)$ were used during vaginal hysterectomy. The surgical procedure was performed with the same technique in both study groups. The primary outcomes were duration of surgery and estimated blood loss. The secondary outcomes were intra-operative complications and post-operative complications.

Results: The duration of surgery and estimated blood loss in the vessel sealing group was significantly less than in the conventional suture group $(p<0.05)$. No significant difference was present between the two groups in the rate of intra-operative and post-operative complications.

Conclusions: The primary outcome of our study is that the EBVS system can be used equally and even more effectively in some aspects; and as safe an alternative approach to conventional suture ligation technique during vaginal hysterectomies performed specifically on morbidly obese patients with reduced operation times and blood loss, and without increasing the complication rates.
\end{abstract}

Key words: vaginal hysterectomy; vessel sealing; morbid obesity

\section{INTRODUCTION}

As reported by the World Health Organization (WHO) in 2008, the prevalance of obesity (body mass index $[\mathrm{BMI}] \geq 30 \mathrm{~kg} / \mathrm{m}^{2}$ ) among Turkish women was estimated at $34 \%$. Among European countries, this level ranks in 4th place after Malta, the Czech Republic, and Slovenia. Obesity is a noncontagious complex disease with unclear etiology. Obese patients are under increased risk of surgical complications and adverse medical outcomes [1].

The American College of Obstetricians and Gynecologists (ACOG) recommended that vaginal hysterectomy $(\mathrm{VH})$ should be preferred over abdominal hysterectomy $(\mathrm{AH})$; when $\mathrm{VH}$ is not feasible, laparoscopic hysterectomy (LH) can avoid the need to perform open surgery [2]. Although $\mathrm{VH}$ has been proven to be superior to other routes of hysterectomy with respect to patient safety, perioperative morbidity and economic and cosmetic factors, other routes are often preferred. The main reasons cited for performing hysterectomy through alternative routes are the surgeon's skill and experience level, as well as the patient's obesity, nulliparity, and the need for concommitant surgeries [3].

Evidence-based absolute contraindications for vaginal hysterectomy include malignancy, an undiagnosed pelvic mass, and inability to access uterine vessels, but not obesity. 
In order to render vaginal hysterectomy a safer and easy to perform operation, there has recently been increased interest in the development of faster, easier, and more efficient hemostatic methods rather than using conventional knot tying for vessel ligation [3-5]. The potential advantages of using electrosurgical bipolar vessel sealing (EBVS) for vaginal hysterectomies have been demonstrated in many studies; however, the efficacy and safety of using such devices in morbidly obese patients has not been studied to the same extent.

\section{OBJECTIVES}

The aim of the present study was to assess the safety and efficacy of EBVS system for vaginal hysterectomy in morbidly obese patients, and to compare the surgical outcomes of this method with the conventional suture ligation technique.

\section{MATERIAL AND METHODS}

\section{Study design. Retrospective cohort analysis. Data collection}

A total of 105 morbidly obese patients who underwent vaginal hysterectomy due to benign gynecologic pathologies between January 2010 and April 2017 were included in the study. The ethics board of Istanbul Health Sciences University Bağcılar Education and Research Hospital approved the study. A policy of evaluating all patients scheduled for hysterectomy as being suitable for vaginal hysterectomy is followed by the urogynecology department of the hospital. According to this policy, patients suspected of having malignancy and/or extrauterine disease, an immobile uterus or a vaginal width less than or equal to 2 fingers during bimanual examination are considered for alternative methods of hysterectomy. The weights and heights of each patient were measured at the clinic and the BMI was calculated as the weight in kilograms divided by the square of height in meters. Patients with a BMI $\geq 40 \mathrm{~kg} / \mathrm{m}^{2}$ (Class III) are defined to be severely or morbidly obese. The WHO criteria were used to determine the menopausal state of patients.

Patients with the following criteria were selected for inclusion in the study from the retrospective data pool: 1) $\mathrm{BMI} \geq 40 \mathrm{~kg} / \mathrm{m}^{2}$; 2) age $\geq 18$ years; 3 ) clinical follow up $\geq 30$ days; and 4) symptomatic second-degree uterine prolapse (Halfway Scoring System). The exclusion criteria for the study were: 1) a past history of urogynecologic surgery; 2) need for concommitant urogynecologic surgery; 3) a postoperative uterine weight of more than $280 \mathrm{~g}$; 4) receiving hormonal therapy; and 5) failure to attend follow-up gynecologic examination during the postoperative first three months.

Patient characteristics including age at the time of surgery, BMI, parity, menopausal status, past surgical history, past medical history, and peri and post-operative outcomes were collected using the international classification database (ICD) codes from the electronic medical database of the hospital. Patients were divided into two groups according to whether conventional suture ligation technique or EBVS system were used during their vaginal hysterectomy.

\section{Intervention}

Preoperative clinical examinations (transvaginal ultrasonography and bimanual examination), human papillomavirus (HPV) DNA test, and Papanicolaou (PAP) smear were performed to exclude cervical pathologies. Patients were hospitalized one day prior to surgery. At the time of anesthesia induction, $2 \mathrm{~g}$ intravenous (IV) cefazolin was administered for antibiotic prophylaxis. In accordance with the recommendations of the American College of Obstetrics and Gynecology and American College of Chest Physicians, moderate risk patients used gradual compression stockings and received $40 \mathrm{mg}$ enoxaparin 2 hours prior to the operation and daily until discharge from hospital. High-risk patients, wore gradual compression stockings and received $40 \mathrm{mg}$ enoxaparin 2 hours before the operation and up to 4 weeks post-operatively.

All vaginal hysterectomies were performed by experienced specialists and trainees under their supervision. To provide adequate exposure, the patient was positioned so that the vaginal introitus was at the edge of the operating table. Patients'legs were positioned using boot-type stirrups (Yellofins ${ }^{\circledR}$ Elite Stirrups with Lift-Assist ${ }^{\mathrm{TM}}$, Allen Medical Systems, Boston, USA). Labia majoras were sutured laterally to expose the vaginal introitus when needed. The bowels were pushed away towards the abdominal cavity using tampon gauzes to prevent thermal damage with EBVS in patients undergoing salpingo-oophorectomy.

The surgical procedure was performed with the same technique in both study groups. A circular incision was made around the cervix. The pouch of Douglas was entered posteriorly and the anterior cul de sac entered anteriorly, followed by separation of the bladder from the uterus. In the control group, ligaments and vessels were clamped, cut, and sutured using no:1 polyglactin 910 braided sutures (Vicryl ${ }^{\oplus}$; Ethicon Inc., Somerville, USA). In the EBVS group (Fig. 1), uterine pedicles were sealed until a signal indicating adequate coagulation was obtained from the electrosurgical generator and then the pedicles were cut (Fig. 2). The EBVS instrument used in this study was the Ligasure Impact $^{\mathrm{TM}}$ (Medtronic, Dublin, Ireland), and the electrosurgical generator was the ForceTriad ${ }^{\mathrm{TM}}$ Energy Platform (Medtronic, Dublin, Ireland). Hemostasis was maintained following the extraction of the uterus from the vagina. The peritoneum and vaginal cuff were approximated using no:1 standard sutures. 


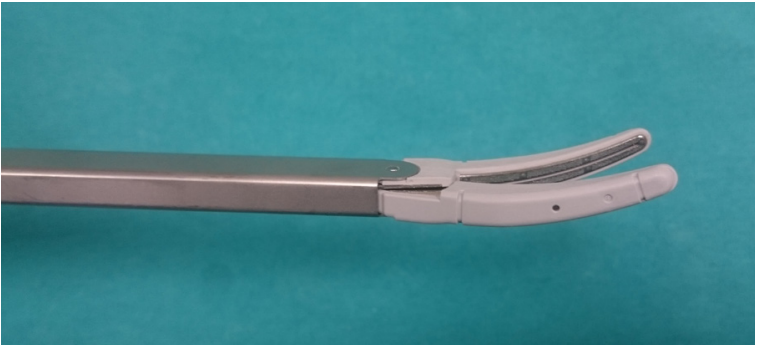

Figure 1. Bipolar vessel sealing system consists of a standart-size Heaney-type clamp and a vessel-sealing generator with a feedback mechanism that provides an audible tone when the seal cycle is complete

The estimated blood loss (EBL) was calculated as the difference between the preoperative and postoperative day 1 hemoglobin levels. The total operation time was calculated from the time an incision was made on the anterior vaginal fornix until the removal of the uterus. The duration of hospitalization was calculated as the number of days between the day of surgery and the day of discharge from hospital. Uterine weights were obtained from the pathology reports. All patients were called for follow-up gynecologic examinations after the $1^{\text {st }}$ week and $3^{\text {rd }}$ month postoperatively.

\section{Statistical analysis}

The data were interpreted and verified by an independent observer. The study data are presented as mean \pm standard deviation and percentages (\%). A comparison of continuous variables with normal distribution was made using the independent-samples (unpaired) Student's t-test and those without normal distribution were evaluated using the Mann-Whitney U test. Fisher's exact test and Pearson $x^{2}$ tests were used to compare nominal and categorical data. All calculations were based on a two-sided hypothesis with a P value of $<0.05$ considered as significant. The Statistical Package for the Social Sciences (SPSS) version 19 was used for statistical analyses (IBM, Armonk, NY, USA).

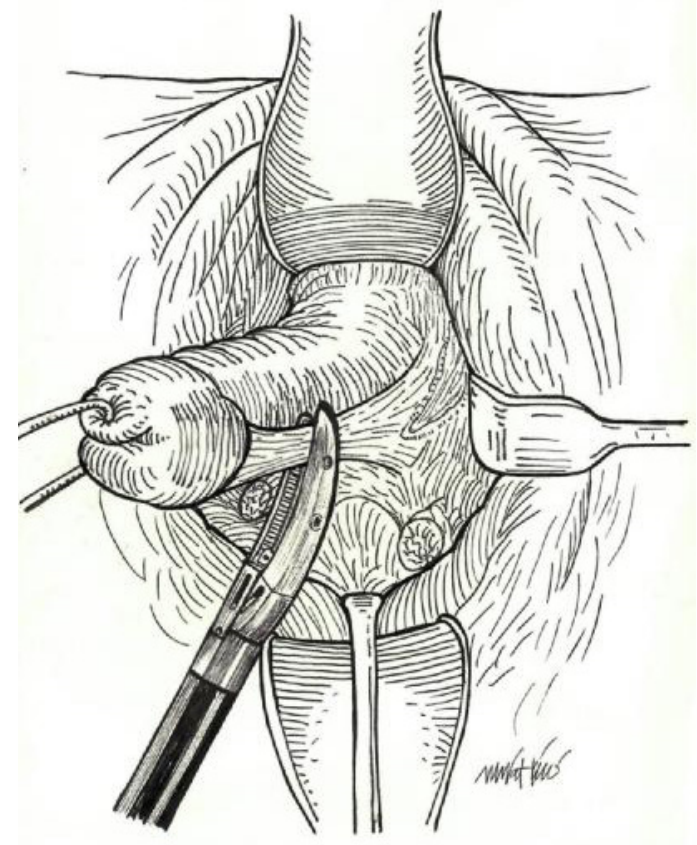

Figure 2. Positioning of the electrosurgical bipolar vessel sealing device

\section{RESULTS}

Of the 105 morbidly obese patients who underwent vaginal hysterectomy, 41 underwent EBVS, and 64 underwent conventional suture ligation technique. No difference was found between the two groups with respect to age, BMl, parity, menopause status, prior surgical history, and medical comorbidities. Patient characteristics in the two groups are presented in Table 1.

The operation time in the EBVS group was significantly shorter than in the conventional suture group $(101.4 \pm 17 \mathrm{~min}$ and $142 \pm 21.4$ min; $p<0.01$, respectively). A $28 \%$ decrease was present in the operation times when EBVS system were used instead of conventional suture ligation. The estimated blood loss was significantly lower in the EBVS group

Table 1. Baseline demographic characteristics of patients

\begin{tabular}{|l|c|c|c|}
\hline & $\begin{array}{c}\text { Conventional suture group } \\
(\mathbf{n}=\mathbf{6 4})\end{array}$ & $\begin{array}{c}\text { Vessel sealing group } \\
(\mathbf{n}=\mathbf{4 1})\end{array}$ & $\mathbf{P}$ \\
\hline Age $($ years) & $49.3 \pm 12.9$ & $51.2 \pm 11.5$ & 0.55 \\
\hline BMl, $\left[\mathrm{kg} / \mathrm{m}^{2}\right]$ & $46.2 \pm 5.08$ & $44.4 \pm 2.7$ & 0.33 \\
\hline Parity & $3.5 \pm 1.4$ & $3.3 \pm 1.2$ & 0.42 \\
\hline Postmenopausal status $\mathrm{n}(\%)$ & $24(37.5)$ & $13(31.7)$ & 0.23 \\
\hline Scarred uterus $\mathrm{n}(\%)$ & $5(7.8)$ & $3(7.3)$ & 0.21 \\
\hline Previous non-uterine procedure $\mathrm{n}(\%)$ & $6(9.3)$ & $4(9.7)$ & 0.68 \\
\hline Medical comorbidity ${ }^{\mathrm{n}} \mathrm{n}(\%)$ & $24(37.5)$ & $18(43.9)$ & 0.43 \\
\hline
\end{tabular}

Data are presented as mean \pm SD unless otherwise noted

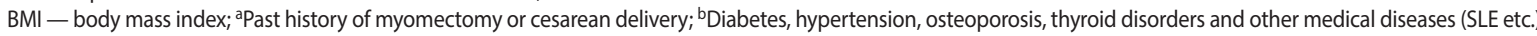


than in the conventional suture group $(3.1 \pm 0.4 \mathrm{~mL} / \mathrm{dL}$ vs. $2.1 \pm 0.9 \mathrm{~mL} / \mathrm{dL} ; \mathrm{p}<0.01$, respectively). No significant difference was present between the two groups in the length of hospital stay $(p=0.41)$. The number of patients who requested salpingo-oophorectomy was 13 in the EBVS group and 18 in the conventional suture group. The successfully performed salphingo-oophorectomy rate was significantly higher in the EBVS group than in the conventional suture group (11/13 (84.6\%) vs. $7 / 18$ (38.8\%), p < 0.01, respectively).

Conversion rates from vaginal hysterectomy to abdominal hysterectomy were not significantly different between the two groups $(p>0.05)$. Three patients in the EBVS group had to be converted to abdominal hysterectomy due to uncontrollable hemorrhage from the infindibulopelvic pedicle. In the conventional suture group, 2 patients underwent conversion to abdominal hysterectomy due to hemorrhage from the infindibulopelvic ligament, and 4 due to inadequate tissue exposure. No significant difference was present between the two groups in the amount of blood transfusions, change in white blood cells (WBC), uterine weight, need for analgesics, and type of anesthesia received $(p>0.05)$. The operative parameters in the two groups are presented in Table 2.

No significant difference was present between the two groups in the rate of intra-operative and post-operative complications. One patient developed arrhythmia immediately following anesthesia induction and was taken to the intensive care unit without undergoing surgery. No bowel or ureteral injury was encountered in either of the study groups. Four patients (6.2\%) in the conventional suture group and 2 patients (4.8\%) in the EBVS group had a urinary bladder injury. All patients with bladder injuries had a history of cesarean delivery. Two patients in the conventional suture group (3.1\%) had to be reoperated on the postoperative first day, and 1 patient (2.4\%) in the EBVS group had to be reoperated on the postoperative $6^{\text {th }}$ day because of vaginal hemorrhage and cuff hematoma. The intraoperative and postoperative complications in the two groups are presented in Table 3.

\section{DISCUSSION}

In our study, the EBVS system improved the surgical outcome by shortening the operative time and decreasing the blood loss without increasing the complication rates compared with conventional suture ligation technique in a retrospectively selected population of morbidly obese patients.

A Cochrane review on surgical approaches to hysterectomy for benign gynecologic diseases states that the vaginal approach has to be considered as the best choice for the uterus removal because of its association with fewer complications, reduced operating time, decreased hospitalization, lower costs, and shorter convalescence compared with $\mathrm{AH}$ [6]. Despite its many demonstrated advantages, $\mathrm{VH}$, a type of natural orifice surgery, accounts for only $23 \%$ of hysterectomies performed in the United States [7]. The route of hysterectomy is frequently chosen according to the experience and ability of the surgeon, uterine weight, history of prior pelvic surgery, and patient characteristics such as obesity [8].

Previous studies have shown $\mathrm{VH}$ to be feasible and safe when performed in obese patients (BMI $\geq 30 \mathrm{~kg} / \mathrm{m}^{2}$ ) with

Table 2. Comparison of surgical outcomes using electrosurgical bipolar vessel sealing and conventional suture ligation technique

\begin{tabular}{|c|c|c|c|}
\hline & $\begin{array}{l}\text { Conventional suture group } \\
\qquad(n=64)\end{array}$ & $\begin{array}{l}\text { Vessel sealing group } \\
\qquad(n=41)\end{array}$ & $\mathbf{P}$ \\
\hline Duration of surgery (min) ${ }^{a}$ & $142 \pm 21.4$ & $101.4 \pm 17$ & $<0.01$ \\
\hline Reduction in hb level [g/dL] & $3.1 \pm 0.4$ & $2.1 \pm 0.9$ & $<0.01$ \\
\hline Blood transfusion $\mathrm{n}(\%)^{\mathrm{b}}$ & $3(4.6)$ & $2(4.8)$ & 0.31 \\
\hline Uterine weight [g] & $102 \pm 70.2$ & $111 \pm 49.3$ & 0.53 \\
\hline WBC level & $5.87 \pm 3.6$ & $5.75 \pm 2.7$ & 0.20 \\
\hline Length of hospitalization (days) & $2.71 \pm 0.9$ & $2.87 \pm 1.2$ & 0.41 \\
\hline Concomitant salpingo-oophorectomy n (\%) & $7(38.8)$ & $11(84.6)$ & $<0.01$ \\
\hline Conversion to laparotomy n (\%) ${ }^{c}$ & $6(9.3)$ & $3(7.3)$ & 0.09 \\
\hline Need for analgesia (days) & $2.14 \pm 0.6$ & $2.0 \pm 0.5$ & 0.14 \\
\hline \multicolumn{4}{|l|}{ Anesthesia n (\%) } \\
\hline General & $15(23.4)$ & $8(19.5)$ & 0.47 \\
\hline Regional (spinal or spinal/epidural) & $49(76.5)$ & $33(80.4)$ & 0.53 \\
\hline
\end{tabular}

Data are presented as mean \pm SD unless otherwise noted;

ancludes the operation time of hysterectomy only; ${ }^{b}$ Preoperatively performed blood transfusions for anemia are not included; ${ }^{c}$ Conversion due to intractable hemorrhage or non-feasibility of the procedure; 


\begin{tabular}{|c|c|c|c|}
\hline & $\begin{array}{l}\text { Conventional suture group } \\
\qquad(n=64)\end{array}$ & $\begin{array}{l}\text { Vessel sealing group } \\
\qquad(\mathrm{n}=41)\end{array}$ & $\mathbf{P}$ \\
\hline \multicolumn{4}{|c|}{ Intra-operative complications n (\%) } \\
\hline Bladder injury & $4(6.2)$ & $2(4.8)$ & NS \\
\hline Ureteral injury & - & - & - \\
\hline Bowel injury & - & - & - \\
\hline Arrhythmia & 0 & $1(2.4)$ & NS \\
\hline \multicolumn{4}{|c|}{ Post-operative complications n (\%) } \\
\hline Vault bleeding/hematoma & $3(4.6)$ & $2(4.8)$ & NS \\
\hline Vault infections/cellulitis & $4(6.2)$ & $2(4.8)$ & NS \\
\hline Urinary tract infections & $8(12.5)$ & $5(12.1)$ & NS \\
\hline Tromboembolic events & - & - & - \\
\hline Pelvic absess & - & - & - \\
\hline Need for re-operation & $2(3.1)$ & $1(2.4)$ & NS \\
\hline Febril events & $6(9.3)$ & $5(12.1)$ & NS \\
\hline Ileus & - & - & - \\
\hline
\end{tabular}

Data are presented as number (percent) unless otherwise noted; NS — non-significant

benign diseases [8-10]. One of the drawbacks of performing $\mathrm{VH}$ is having to reach vascular pedicles deep in the pelvis through a limited amount of space, which may be more pronounced in patients who are morbidly obese. Characteristics that limit exposure of the surgical site such as prominent buttocks, redundant vaginal tissue, and decreased soft tissue pliability may complicate the surgical procedure or even prevent it from being performed all together [11]. Bogani et al pointed out that the patient BMI is directly proportional to the rate of complications due to hysterectomy. The higher intraoperative complication rates in our study and control groups compared with those reported in previously published studies could be explained by these factors [9, 12, 14-17].

Providing new technical improvements that make vascular clamping less difficult could increase the rates of $\mathrm{VH}$ in challenging cases. Accordingly, a few authors suggested the use of advanced electrosurgical instruments that provide the ability to rapidly and sequentially seal vessels and coagulate and transect pedicles with a tool manipulated by one hand. Previous studies showed such instruments adapted to vaginal surgery from laparoscopic practice were suitable alternatives to conventional suturing techniques and had similar safety profiles $[7,12,13]$. To our knowledge, no studies have yet compared the surgery-related outcomes of EBVS system and conventional suture ligation technique only focusing on the morbidly obese patients undergoing $\mathrm{VH}$. Although there are numerous papers on the technique of sealing vessels using bipolar electrosurgical device during $\mathrm{VH}$ also in obese patients, previous studies comparing the two techniques excluded or did not report specifically findings regarding morbidly obese patients. [11, 14-17]. There is no reliable information regarding the impact of EBVS system on surgical success in morbidly obese patients undergoing vaginal hysterectomy.

Similar to studies by Elhao and Gizzo et al., involving non-obese patients, operation times were found in the present study to be significantly reduced with the use of EBVS in obese patients [17]. In our study, a 28\% decrease was demonstrated in operation times when vessel sealing devices were used instead of conventional suturing techniques. The ability of these devices to reduce the duration of operations seems to be more pronounced in the morbidly obese. While operating morbidly obese patients, it is difficult to visualize the surgical field; and accurate stitching and needle retrieval can be problematic. As Ding et al. suggested, EBVS may prevent problems arising from slippage or dislodgement of sutures, thus decreasing the possibility of hemorrhage [11]. Operation times may be reduced when using vessel sealing devices because no time is spent suturing knots and hemorrhage from small vessels within the surgical site is prevented.

A common opinion among authors is that one of the main challenging steps in $\mathrm{VH}$ is the removal of the ovaries. In the presence of adnexial pathologies as the indication or the coindication of hysterectomy, the abdominal route is more commonly preferred. The cases who are planned to be operated through the vaginal route were those without a primary adnexial pathology. The salpingo-oophorectomies performed for these women were mainly due to the patients' requests [18]. Eventually, we compared the technically achieved salpingoofectomies through the vaginal route in the EBVS and the conventional suturing groups. In 
the present study, the rate of salphingo-oophorectomy in the EBVS group was approximately $45 \%$ higher than that of the conventional suture group. These results, in accordance with the literature, suggest that it appears safe to use EBVS in morbidly obese patients to coagulate vessels that are difficult to reach and suture with conventional technique [19].

One of the most important strengths of this study was its design, addressing surgical outcomes specifically in morbidly obese patients who underwent surgery without urogynecologic procedures. The main limitation of this retrospective study, however, is that the surgical approach to be used was determined by the individual surgeon. Confounding by indication cannot be eliminated from the present study because the surgeon's level of expertise is one of the factors affecting the choice of surgery. Although all hysterectomies were performed by the same surgical team, which comprised expert surgeons, the number of trainees who actively participated could not be determined for certain. However, we believe the number of trainees involved in the surgical treatment of the morbidly obese patients was small because they are a more difficult and challenging patient population. Nevertheless, a difference of approximately 40 minutes in the operation times between the two groups is believed to be due largely to the surgical procedure rather than the performance of the surgeons involved. Another limitation of this study is the small sample size, which warrants further confirmation in larger scale studies.

It is evident that the surgical management of obese patients will continue to be a challenge for gynecologic surgeons. EBVS system, which have been described by Hefni et al. as instruments that can be easily operated independently of the skill of the surgeon, can help improve surgical results and widen indications in morbidly obese patients [20]. Furthermore, as cited in recent studies, it may be interesting to compare the surgical results, especially the amount of blood loss, of vaginal hysterectomies performed using EBVS systems and laparoscopic hysterectomies in future randomized controlled trials [9].

\section{CONCLUSIONS}

The primary outcome of our study is that the EBVS system can be used equally and even more effectively in some aspects; and as safe an alternative approach to conventional suture ligation technique during vaginal hysterectomies performed specifically on morbidly obese patients with reduced operation times and blood loss, and without increasing the complication rates.

\section{Acknowledgments}

We would like to thank Lale Susan Wetherilt M.D. Asst. Prof. and David F. Chapman Bsc for editing the article.

\section{Conflict of interest}

The authors have no conflicts of interest or financial ties to disclose.

\section{REFERENCES}

1. World Health Organization. Country profiles on nutrition, physical activity and obesity in the 53 WHO European Region Member States. WHO website. http://www euro who int/_ data/assets/pdf file/0004/243337/Summary-document-53-MS-country-profile pdf (2013 07).

2. ACOG Committee Opinion No. 444: choosing the route of hysterectomy for benign disease. Obstet Gynecol. 2009; 114(5): 1156-1158, doi: 10.1097/AOG.0b013e3181c33c72, indexed in Pubmed: 20168127.

3. Muffly TM, Kow NS. Effect of obesity on patients undergoing vaginal hysterectomy. J Minim Invasive Gynecol. 2014; 21(2): 168-175, doi: 10.1016/j.jmig.2013.07.017, indexed in Pubmed: 23933353.

4. Harmanli $\mathrm{OH}$, Dandolu V, Isik EF, et al. Does obesity affect the vaginal hysterectomy outcomes? Arch Gynecol Obstet. 2011; 283(4): 795-798, doi: 10.1007/s00404-010-1422-4, indexed in Pubmed: 20237934.

5. Sheth SS. Vaginal Hysterectomy. 2nd ed. Jaypee Brothers (UK) Medical Publishers LTD. ; 2014.

6. Nieboer TE, Johnson N, Tavender E, et al. Surgical approach to hysterectomy for benign gynaecological disease. Cochrane Database Syst Rev. 2009(3): CD003677, doi: 10.1002/14651858.CD003677.pub4, indexed in Pubmed: 19588344

7. Cronjé HS, de Coning EC. Electrosurgical bipolar vessel sealing during vaginal hysterectomy. Int J Gynaecol Obstet. 2005; 91(3): 243-245, doi: 10.1016/j.ijgo.2005.08.019, indexed in Pubmed: 16243338.

8. Rafii A, Samain E, Levardon M, et al. Vaginal hysterectomy for benign disorders in obese women: a prospective study. BJOG. 2005; 112(2): 223-227, doi: 10.1111/j.1471-0528.2005.00563.x, indexed in Pubmed: 15663588.

9. Bogani G, Cromi A, Serati M, et al. Laparoscopic and vaginal approaches to hysterectomy in the obese. Eur J Obstet Gynecol Reprod Biol. 2015; 189: 85-90, doi: 10.1016/j.ejogrb.2015.02.035, indexed in Pubmed: 25898369.

10. Sheth SS. Vaginal hysterectomy as a primary route for morbidly obese women. Acta Obstet Gynecol Scand. 2010; 89(7): 971-974, doi: 10.3109/00016341003681256, indexed in Pubmed: 20199359.

11. Ding Z, Wable $M$, Rane A. Use of Ligasure bipolar diathermy system in vaginal hysterectomy. J Obstet Gynaecol. 2005; 25(1): 49-51, doi: 10.1080/01443610400024609, indexed in Pubmed: 16147695.

12. Zubke W, Hornung R, Wässerer $S$, et al. Bipolar coagulation with the BiClamp forceps versus conventional suture ligation: a multicenter randomized controlled trial in 175 vaginal hysterectomy patients. Arch Gynecol Obstet. 2009; 280(5): 753-760, doi: 10.1007/s00404-009-1010-7, indexed in Pubmed: 19252920

13. Kroft J, Selk A. Energy-based vessel sealing in vaginal hysterectomy: a systematic review and meta-analysis. Obstet Gynecol. 2011; 118(5): 1127-1136, doi: 10.1097/AOG.0b013e3182324306, indexed in Pubmed: 22015881.

14. Samulak D, Wilczak M, Michalska MM, et al. Vaginal hysterectomy with bipolar coagulation forceps (BiClamp) as an alternative to the conventional technique. Arch Gynecol Obstet. 2011; 284(1): 145-149, doi: 10.1007/s00404-010-1617-8, indexed in Pubmed: 20683602.

15. Silva-Filho AL, Rodrigues AM, Vale de Castro Monteiro M, et al. Randomized study of bipolar vessel sealing system versus conventional suture ligature for vaginal hysterectomy. Eur J Obstet Gynecol Reprod Biol. 2009; 146(2): 200-203, doi: 10.1016/j.ejogrb.2009.03.014, indexed in Pubmed: 19380188.

16. Lakeman MME, The S, Schellart RP, et al. Electrosurgical bipolar vessel sealing versus conventional clamping and suturing for vaginal hysterectomy: a randomised controlled trial. BJOG. 2012; 119(12): 1473-1482, doi: 10.1111/j.1471-0528.2012.03484.x, indexed in Pubmed: 22925365. 
17. Elhao M, Abdallah K, Serag I, et al. Efficacy of using electrosurgical bipolar vessel sealing during vaginal hysterectomy in patients with different degrees of operative difficulty: a randomised controlled trial. Eur J Obstet Gynecol Reprod Biol. 2009; 147(1): 86-90, doi: 10.1016/j. ejogrb.2009.07.011, indexed in Pubmed: 19729238.

18. ACOG CommitteeOpinion No. 578: Elective surgery and patient choice. Obstet Gynecol. 2013; 122(5): 1134-1138, doi: 10.1097/01. AOG.0000437384.88715.03, indexed in Pubmed: 24150029.
19. Davies A, Vizza $E$, Bournas N, et al. How to increase the proportion of hysterectomies performed vaginally. Am J Obstet Gynecol. 1998; 179(4): 1008-1012, doi: 10.1016/s0002-9378(98)70207-8, indexed in Pubmed: 9790389.

20. Hefni MA, Bhaumik J, El-Toukhy T, et al. Safety and efficacy of using the LigaSure vessel sealing system for securing the pedicles in vaginal hysterectomy: randomised controlled trial. BJOG. 2005; 112(3): 329-333, doi: 10.1111/j.1471-0528.2004.00325.x, indexed in Pubmed: 15713149. 